\title{
Thyroid hormones increase collagen I and cartilage oligomeric matrix protein (COMP) expression in vitro human tenocytes
}

\author{
Anna C. Berardi 1 \\ Francesco Oliva ${ }^{2}$ \\ Martina Berardocco ${ }^{1}$ \\ Marina la Rovere ${ }^{1}$ \\ Patrizia Accorsi ${ }^{3}$ \\ Nicola Maffulli ${ }^{4}$
}

1 UOC Immunohematology and Transfusion Medicine Laboratories, Laboratory of Stem Cells, Spirito Santo Hospital, Pescara, Italy

2 Department of Orthopedics and Traumatology, University of Rome "Tor Vergata", School of Medicine, Rome, Italy

3 UOC Immunohematology and Transfusion Medicine Laboratories, Spirito Santo Hospital, Pescara, Italy

4 Department of Musculoskeletal Disorders, Faculty of Medicine and Surgery, University of Salerno, Salerno, Italy; and Queen Mary University of London, Barts and The London School of Medicine and Dentistry, Institute of Health Sciences Education, Centre for Sports and Exercise, London, UK

Corresponding author:

Anna C. Berardi

UOC Immunohematology and Transfusion Medicine Laboratories, Laboratory of Stem Cells, Spirito Santo Hospital, Pescara, Italy

E-mail: annac.berardi@ausl.pe.it; annacberardi@yahoo.it

\section{Summary}

Background: we previously demonstrated the presence of high levels of thyroid hormones (THs) receptors isoforms in healthy tendons, their protective action during tenocyte apoptosis, and the capability to enhance tenocyte proliferation in vitro. In the present study we tested the ability of THs to influence ECM protein tenocyte secretion in an in vitro system.

Methods: primary tenocyte-like cells were cultivated for 1,7 and 14 days in the presence of $T_{3}$ or $\mathrm{T}_{4}$ individually or in combination with ascorbic acid (AA).

Results: $\mathrm{THs}\left(\mathrm{T}_{3}\right.$ or $\left.\mathrm{T}_{4}\right)$ in synergism with AA increase significantly the total collagen production after 14 days. THs in synergism with AA increase significantly the expression of collagen I,biglycan and COMP, after some days.

Conclusion: THs play a role on the extra cellular matrix of tendons, enhancing in vitro the production of several proteins such as collagen I, biglycan and COMP. THs receptors are active on human tenocytes, and can play a role in tendon ailments.

KEY WORDS: ascorbic acid, collagen I, COMP, tenocytes, thyroid hormones, tendons.

\section{Introduction}

Tendinopathies and tendon ruptures are the most common musculoskeletal disorders with high social costs. Metabolic disorders (e.g. diabetes, hypercholesterolemia, hyperuricaemia and obesity) and congenital metabolic disorders are an emerging co-factors involved in the pathogenesis of tendon diseases ${ }^{1,2}$. The potential role of thyroid hormones during tendons diseases has been investigated only for calcific tendinopathy of rotator cuff 3,4 .

Tendons are fibrous connective tissues that are composed of cells within a complex extra cellular matrix (ECM) rich of collagens, proteoglycans, glycoprotein and water ${ }^{5}$. The fibril-forming type I collagen is the major component responsible for the tensile strength of the ECM. Between and within the type I collagen fibers are other important matrix molecules such as type III and V collagens, that contains proteoglycans (PGs) and glycoproteins. The PGs (decorin, biglycan, fibromodulin, etc.) are anionic molecules that have collagen-binding proprieties and interact with the collagen fibers and other matrix molecules, regulating the ECM assembly, including fibrillogenesis 6,7 .

COMP (Cartilage Oligomeric Matrix Protein) or Trombospondin 5, first identified in cartilage, is an abundant glycoprotein particularly present in tendon exposed to compressive load. COMP belongs to the thrombospodin gene family with the ability to bind to type I, II, and IX collagen molecules as well as fibronectin. COMP acts as a catalyst in collagen fibrillogenesis in vitro, and there are also indications that COMP acts as a regulator in vivo in the extracellular fibril assembly 8 -15.

The essential role of $\mathrm{THs}, \mathrm{T}_{3}$ (triiodothyronine) and $\mathrm{T}_{4}$ (thyroxine) in the development and metabolism of many tissues and organs, both in early and adult life, is mediated mainly through $T_{3}$, which regulates gene 
expression by binding to the $\mathrm{TH}$ receptors (TR)- $\alpha$ and $-\beta$. Thyroid hormones have been reported to modulate cells morphology, differentiation, and proliferation $^{16-18}$, and to regulate ECM organization and synthesis ${ }^{19-24}$. We recently showed that thyroid receptors (TRa/ $\beta$ isoforms) are present on rotator cuff tendons, that THs impacts favorably on the growth of human healthy tenocytes in vitro culture in a dose dependent manner, and that THs protect from apoptosis human healthy tenocytes isolated from human tendon, again in a dose dependent manner 25 .

Theoretically hyperthyroidism may be accompanied by increased catabolism of both soluble and insoluble collagen. Hypothyroidism seems to be accompanied by decreased rates of catabolism of collagen ${ }^{26-30}$.

However, despite these observations, the effects of thyroid hormones on the biosynthesis of ECM proteins by the tenocytes have not yet systematically studied. In the present study we tested the ability of THs to influence ECM protein tenocyte secretion in an in vitro system.

\section{Materials and methods}

\section{Patients and methods}

All the procedures described in this investigation were approved by our local ethics committee. All the patients gave written informed consent to be included in this study. Tendon samples were harvested from healthy supraspinatus tendons biopsy specimen in 5 patients who were operated arthroscopically for shoulder instability ( 3 men, 2 women, age: $28 \pm 5$ year). Systemic conditions such as thyroid disorders, diabetes, gynecological condition, neoplasia, rheumatic diseases, epilepsy and any previous or concomitant rotator cuff disease were considered exclusion criteria.

\section{Tendon cell cultures}

Primary human tendon derived cell cultures were established as previously described 25 . In brief, cells were isolated from tissue samples by washing several times with phosphate buffered saline Dulbecco's W/O $\mathrm{Ca}$ and $\mathrm{Mg}(\mathrm{PBS})+1 \%$ penicillin/streptomycin (Invitrogen, Life Technologies, Carlsbad, CA, USA). Small pieces of fresh tendon were carefully dissected and mechanically disaggregated with the aid of fine watchmaker forceps to maximize the interface between tissue and medium. The tendons were immediately placed on Petri dishes of $60 \mathrm{~mm}$ in diameter (Greiner CELLSTAR dish, Sigma-Aldrich, Saint Louis, MO, USA), containing $5 \mathrm{~mL}$ of a-MEM supplemented with $20 \%$ heat-inactivated foetal calf serum (FCS) and $1 \%$ L-glutamine and $1 \%$ penicillin/streptomycin (Gibco, Invitrogen, Life Technologies) at $37^{\circ} \mathrm{C}$ in $5 \%$ $\mathrm{CO}_{2}$ and air with a change of medium every 2-3 days. Tenocytes were then harvested by Stem Pro Accutase (Life Technologies Carlsbad, CA, USA) and centrifugation at $1,500 \mathrm{rpm}$ for $5 \mathrm{~min}$ when the cells mi- grated out of tendon pieces, and reached $60-80 \%$ of confluence (19 days). Collected tenocytes were immediately used for culture to avoid phenotype drift with further in vitro passages ${ }^{31}$. The tenocyte phenotype was confirmed by assessing the expression of a tenocyte-specific gene (scleraxis) and genes for collagens a1(I), a2(I) and $a 1(\mathrm{III})$ in real-time PCR assays with specific primers (data not shown).

The tenocyte-like cells were seeded with $5 \times 10^{3}$ vital cells per well in a 24-well plate (Greiner CELLSTAR dish, Sigma-Aldrich), and in triplicates. Cells were cultured in alpha-MEM supplemented with $1 \%$ L-glutamine and $1 \%$ penicillin/streptomycin and $20 \%$ FBS. Every 3 days, $50 \%$ of the medium was changed. After $24 \mathrm{~h}$, the cultured cells were exposed to two different doses of $T_{3}$ or $T_{4}\left(10^{-7}\right.$ and $\left.10^{-6} \mathrm{M}\right)$ with or without $\mathrm{L}$ ascorbic acid $\left(10^{-7} \mathrm{M}\right)$ (AA) (Sigma Aldrich). Fresh aliquots of $\mathrm{T}_{3}$ or $\mathrm{T}_{4}$ were added each $24 \mathrm{~h}$, while $A A$ was added every 3 days. At day 1, 7 and 14 after seeding, a Sirius red assay was performed to analyze total collagen production.

\section{Picrosirius red staining}

The quantification of total collagen tenocyte-seeded 24-well culture was assessed after 1, 7 and 14 days of incubation as previously described using a modification of the method described by Lopez-de Leon and Rojkind ${ }^{32}$. At the end of $\mathrm{TH}$ exposure, cells were fixed with Bouin's solution [acetic acid 5\% (Sigma Aldrich), formaldehyde $9 \%$ (ICN Biomedicals Inc., Ohio USA), picric acid 0.9\% (Carlo Erba Reagents, Chaussée du Vexin, France)], for 1 hour at room temperature. After fixation, the cell layers were stained with $0.1 \%$ Sirius red-Direct Red 80 in saturated picric acid for $1 \mathrm{~h}$, after which excess Sirius red was removed by washing under running tap water. The dye was then eluted with $0.1 \mathrm{~N} \mathrm{NaOH} /$ methanol (1:1) (Sigma-Aldrich), and the collagen quantified by spectrophotometry at $540 \mathrm{~nm}$. The values were then normalized against total protein concentration. All assays were performed in triplicate. Tenocyte-like cells culture without any treatment were used as control.

\section{Immunofluorescence staining}

The primary tenocyte-like cells were seeded with $5 \times 10^{3}$ vital cells per well in a 2 -well chamber slides (Thermo Fisher Scientific, Inc., Rochester, NY, USA), in triplicates and cultured as previous described. After 1,7 and 14 days of culture the tenocytes were fixed with pure acetone for $10 \mathrm{~min}$ at $-20^{\circ} \mathrm{C}$. Then, washed a few minutes with PBS. Cells were incubated for $30 \mathrm{~min}$ at room temperature with PBS containing 5\% of Bovine Serum Albumin (BSA) (Kedrion Group S.P.A., Lucca, Italy) for protein blockage. Primary antibodies for Anti-type I (1:2000), Anti-type III (1:500) (Sigma-Aldrich), anti-type $\mathrm{V}$ collagen molecules (1:500) (Chemicon International, Inc., Temecula, California, USA), anti-Biglycan (1:100), anti-COMP 


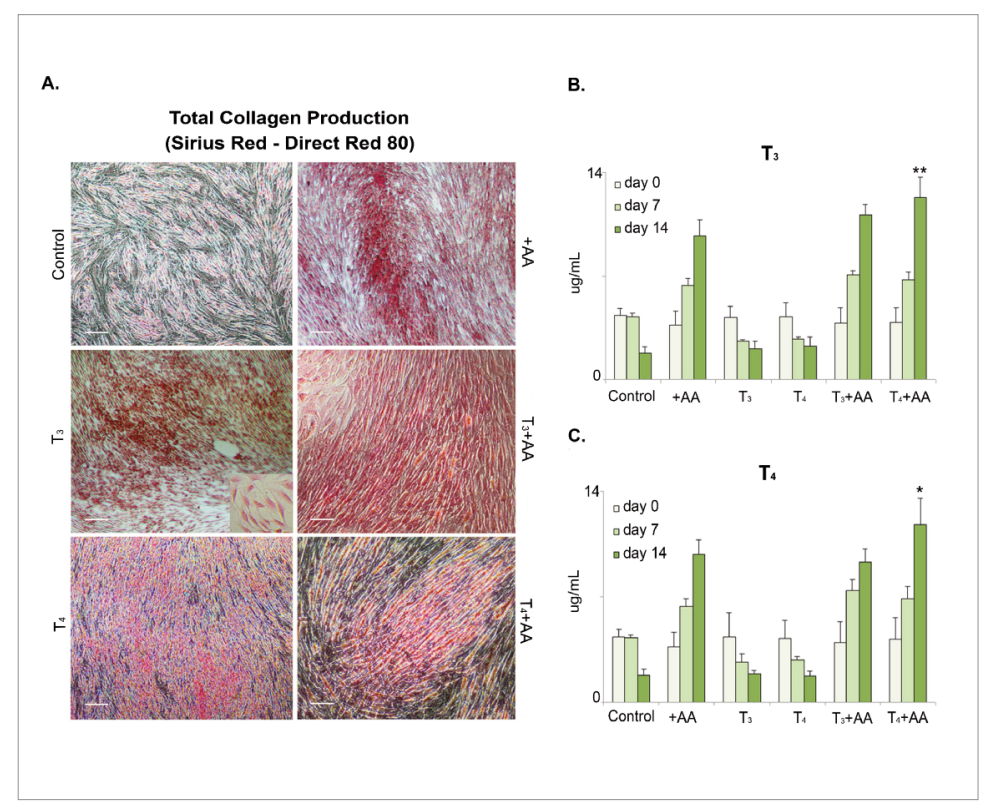

Figure 1. Total collagens production of primary tenoyte-like cells in vitro culture. A) primary tenocyte-like cells isolated from 5 healthy patients, were cultivated for 1,7 and 14 days in the presence of T3 or T4 or T3+ AA and/or T4+AA as control, we used cells without any treatment and cells in the presence of AA were used as positive control for the secretion and accumulation of collagen from primary tenocyte-like cells. Red color show the accumulation of total collagen in the 24 well plates after 14 days of culture followed by Sirius red staining; B), C) quantification of total collagen after 1,7 and 14 days of in vitro culture in the presence of T3 or T4. The collagen was quantified by spectrophotometry at $540 \mathrm{~nm}$. Representative images from 8 independent experiments. The data are expressed as mean \pm SD for 8 independent experiments for samples run in triplicate. $\left(n=8,{ }^{\star} P\right.$ $\left.<0.05,{ }^{*} P<0.01\right)$. Scale $\operatorname{bar}(\mathrm{A}): 50 \mu \mathrm{m}$.

(1:1000), anti-Fibromodulin (1:200) (Abcam, Cambridge UK) and secondary antibodies were diluted in $5 \%$ BSA. Cells were incubated overnight at $4^{\circ} \mathrm{C}$ with primary antibodies, $1 \mathrm{~h}$ with the appropriate fluorochrome antibody at room temperature and then washed a few times with PBS containing 5\% BSA. Molecule's staining Alexa Fluor 488 (Life Tecnologies) was used for type Icollagen and Alexa Fluor 568 (Life Tecnologies) for type Illcollagen, PE was used for type $V$ collagen (Serotec, Oxford UK) and FITC (Sigma Aldrich) for anti-Byclican and anti-COMP staining. After washing with PBS plus 5\% BSA, slides were mounted with $25 \mu \mathrm{L}$ VECTASHIELD ${ }^{\circledR}$ Hard Set Mounting Medium and then were examinated with ECLIPSE Ti-U inverted, fluorescent microscope (Nikon Instruments INC., Melville, NY, USA). Digital images were captured with NIS-Elements Imaging Software (Nikon Instruments INC.).

\section{Statistical Analysis}

Data are typical results from a minimum of three replicated experiments and are expressed as mean \pm SD. Comparison of individual treatment was conducted using Student's $t$ test. Statistical significance in comparison with the corresponding control values was indicated by * $\mathrm{P}<0.05$. versus control; ${ }^{* *} \mathrm{P}<0.01$ versus control.

\section{Results}

\section{Total Collagen production}

In the first series of experiments, we examined whether THs may play a role in the induction of the total collagen production from the primary tenocyte-like cells. Furthermore, as AA regulates collagen synthesis, ${ }^{33,34}$ we used AA individually as positive control, and in combination with $T_{3}$ or $T_{4}$. When $T_{3}$ or $T_{4}$ were used in combination with $\mathrm{AA}$, collagen production of the primary tenocyte-like cells seeded in the 24 wells demonstrated a statistically significant progressively increase after 1,7 and 14 days of culture $\left(n=8,{ }^{*} P\right.$ $<0.05,{ }^{* \star} P<0.01$ ), while, in the presence of $T_{3}$ or $T_{4}$ individually, there was no collagen production, compared with control cells and $+\mathrm{AA}$ positive control (Fig. $1 \mathrm{~A}, \mathrm{~B}, \mathrm{C})$. In addition, $\mathrm{T}_{3}+\mathrm{AA}$ or $\mathrm{T}_{4}+\mathrm{AA}$ produced an increase in total collagen production in a dose-dependent manner. For example, at the dose of $10^{-7} \mathrm{M}$ of $T_{3}$ plus AA in the culture cells, the production of total collagen was $11,14 \pm 1.5 \mu \mathrm{g} / \mathrm{ml}$ compared with $9,75 \pm 1.6$ $\mu \mathrm{g} / \mathrm{ml}$ of AA culture cells. Greater differences were observed at the dose of $10^{-6} \mathrm{M}(12,5 \pm 1.2 \mu \mathrm{g} / \mathrm{ml}$ of $\mathrm{T}_{3}+\mathrm{AA}$ culture vs $9,75 \pm 1,6 \mu \mathrm{g} / \mathrm{ml}$ of AA culture cells). The higher increase was obtained by adding $\mathrm{T}_{3}+\mathrm{AA}$ vs $\mathrm{T}_{4}+\mathrm{AA}\left(10^{-6} \mathrm{M}: 12,5 \pm 1.2 \mu \mathrm{g} / \mathrm{ml}\right.$ vs $\left.11,8 \pm 1.8 \mu \mathrm{g} / \mathrm{ml}\right)$ $\left(n=4,{ }^{\star} P<0.05,{ }^{*} P<0.01\right)$ (Fig. $\left.1 \mathrm{~B}, \mathrm{C}\right)$.

\section{T3 and T4 in synergism with AA modulate the ex- pression of type I Collagen}

Next, we determined and quantified the type of collagen deposed from primary tenocyte-like cells after stimulation with $\mathrm{T}_{3}$ or $\mathrm{T}_{4}$ alone and or in combination of AA. Collagen accumulation was evaluated by immunofluorescence staining of cells cultured on chamber slides. Primary tenocyte-like cells revealed a high amount of collagen I expression (Fig. 2A, B with $T_{3}+A A$ and $\left.T_{4}+A A\right)$. Furthermore, the expression of collagen I was higher in $T_{3}+A A$ than $T_{4}+A A$, and was significantly higher compared to $A A$ used as a control (Fig. 2 B). No presence of collagen III was demonstrated in any culture conditions (Fig. 2 C). Primary 
A.

B.

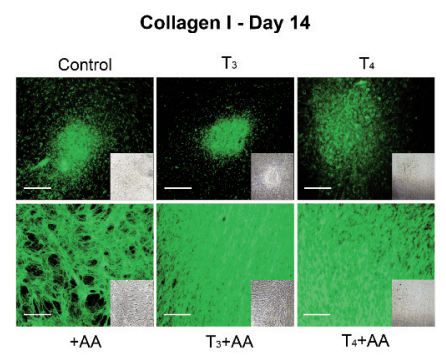

c.

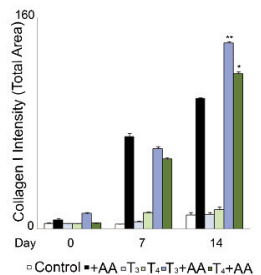

E.

D.

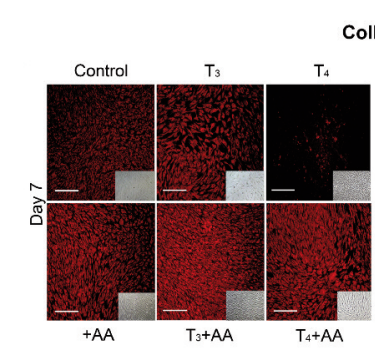

Collagen $\mathrm{V}$

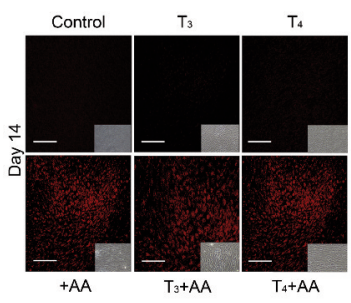

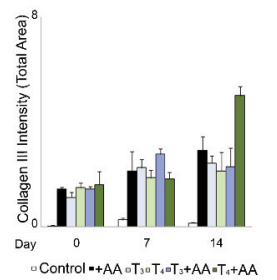

F. tenocyte-like cells showed similar collagen $\mathrm{V}$ expression in all culture conditions, except control cells. Immunofluorescence staining at day 7 reveal any deposition of collagen $\mathrm{V}$ from the primary tenocyte-like cells but only an intracytoplasmic staining. (Fig. 2 D, $\mathrm{F})$. Moreover, at day 14 the expression of collagen $\mathrm{V}$ decreased drastically in $T_{3}+A A, T_{4}+A A$ and control $A A$, and no staining was detected in all the other experimental conditions (Fig. 2 E, F).

\section{Fibromodulin, Biglycan and COMP expression}

Non-collagenous proteins such as fibromodulin, byglican and COMP are present within tendon and play an important role ${ }^{35}$. Immunofluorescence staining of primary tenocyte-like cells cultured on chamber slides revealed no expression of fibromodulin in any of the experimental conditions (data not shown). Moreover, after 14 day of culture in the presence of both $T_{3}+A A$ or $\mathrm{T}_{4}+\mathrm{AA}$, the cells showed low expression of biglycan; this was higher when $T_{3}+A A$ was added to the cells culture (Fig. $3 \mathrm{~A}$ ). COMP expression was presents after 14 day of culture in all culture condition for exception of control cells (Fig. 3 B). In addition, evaluating the amount of immunofluorescence staining through the NIS-Elements Imaging Software, there was a significantly higher intensity in the presence of both $T_{3}+A A$ and $T_{4}+A A$. This was more significant with $\mathrm{T}_{3}+\mathrm{AA}\left({ }^{\star} P<0.05,{ }^{\star *} P<0.01\right)^{36}$ (Fig. $3 \mathrm{C}$ ).

\section{Discussion}

In the present work, we provide evidence that THstreated tenocytes modulate ECM proteins secretion in
Figure 2. Collagen I, III and V expression of primary tenoyte-like cells in vitro culture isolated from 5 healthy patients, and stained as described in material and methods, section $I \mathrm{~m}$ munofluorescence Staining. Representative images from 4 independent experiments. A) Expression of Collagen I after 14 days of culture in green fluorescence and in natural light, showing the primary tenocytelike cells. B) Collagen I Intensity (Total Area was quantified by anticollagen I) it was measured by Nikon software. C) Collagen III Intensity (Total Area). D) Expression of Collagen $\mathrm{V}$ after 7 and 14 days of culture in red fluorescence and in regular light image showing the primary tenocyte-like cells. E) Collagen V Intensity (Total Area). F) Data are expressed as mean \pm SD for 4 independent experiments for samples run in triplicate $\left(n=4,{ }^{\star} P<0.05,{ }^{* \star} P\right.$ $<0.01)$. Scale bar(A, D, E): $50 \mu \mathrm{m}$. vitro. THs treatment, in synergism with $A A$, increases the production of collagen I, Byglican and COMP. The present findings, together with those we previously reported ${ }^{25}$, suggest a binary role for THs on tendons, directly, on tenocyte proliferation, and in synergism with other factors such as AA on tenocyte ECM protein recreations.

Collagen metabolism have been reported to be affected by $\mathrm{THs}^{37,38}$. However, the effect of THs on expression of collagen in human tenocytes had not been previously reported. THs stimulate the synthesis of total collagen in a dose dependent manner during 14 days of culture. The expression of collagen I, the major fibrillar collagen of the tendon, is significantly increased in the primary tenocyte-like cells in vitro after 14 days of culture. This increase of expression leads to collagen synthesis and accumulation. In contrast THs do not affect the expression of collagen III that normally is less abundant in tendon, and increase only during early phase of remodeling ${ }^{39}$ and in tendinopathic tendons ${ }^{40}$. The lack of collagen III productions under TH stress should be considered a protective factors for tendons.

THs in synergism with AA increases the intracytoplasmic expression of collagen $V$ after 7 days of culture to decrease drastically after 14 days. Collagen $\mathrm{V}$ it is known to regulate the characteristic of fibrillar structure in tendon ${ }^{39,41}$.

While the most abundant protein in tendons is collagen I, PGs, comprising $1-5 \%$ of tendon dry weight, are extracellular matrix proteins that play an important role in collagen fibril formation and in resisting compressive loads ${ }^{42-45}$. Biglycan and fibromodulin are $\mathrm{PGs}$ expressed in tendon. Biglycan is a small leucinerich proteoglycan; to date, its role is largely unknown; it is present in the short-term repair response to injury 
A.

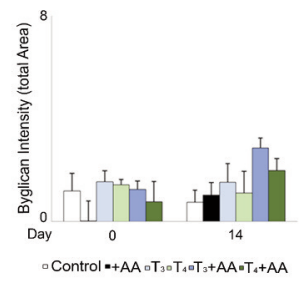

Figure 3. Biglycan and COMP expression of primary tenoyte-like cells in vitro culture. A) Byglican Intensity (Total Area) after 1 and 14 days of culture. $B$ ) Expression of COMP after 14 days of culture in green fluorescence and in regular light image showing the primary tenocyte-like cells. C) COMP Intensity (Total Area). Representative results from 5 independent experiments. Data are expressed as mean \pm SD for 5 independent experiments for samples run in triplicate. $\left({ }^{\star} P\right.$ $\left.<0.05,{ }^{* *} P<0.01\right)$. Scale bar(B): $50 \mu \mathrm{m}$.
B.

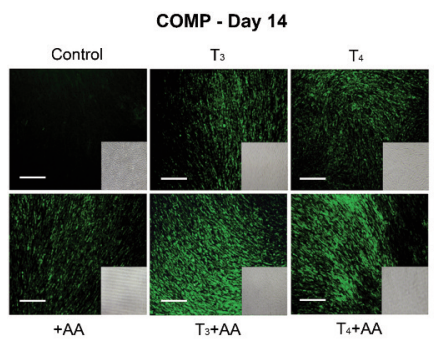

c.

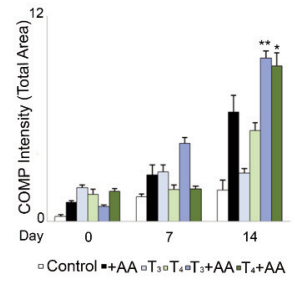

\section{Conflict of interest}

The authors declare no conflict of interest.

\section{Acknowledgements}

The laboratory "Stem Cells" is supported by Francesco Oliva and Laura Bassi ONLUS.

\section{References}

1. de Oliveira RR, Martins CS, Rocha YR, et al. Experimental diabetes induces structural, inflammatory and vascular changes of Achilles tendons. PLoS One. 2013;8(10).

2. Ji JH, Shafi M, Moon CY, Park SE, Kim YJ, Kim SE. Arthroscopic suture bridge technique for intratendinous tear of rotator cuff in chronically painful calcific tendinitis of the shoulder. Orthop Surg. 2013;5(4):289-92.

3. Harvie P, Pollard TC, Carr AJ. Calcific tendinitis: natural history and association with endocrine disorders. J Shoulder Elbow Surg. 2007;16(2):169-73.

4. Oliva F, Barisani D, Grasso A, Maffulli N. Gene expression analysis in calcific tendinopathy of the rotator cuff. Eur Cell Mater. 2011;21:548-57.

5. Tresoldi I, Oliva F, Benvenuto M, Fantini M, Masuelli L, Bei R, Modesti A. Tendon's ultrastructure. Muscles Ligaments Tendons J. 2013;3(1):2-6.

6. Svensson L, Aszódi A, Reinholt FP, Fässler R, Heinegård D, Oldberg Å. Fibromodulin-null mice have abnormal collagen fibrils, tissue organization, and altered lumican deposition in tendon. J Biol Chem. 1999;274:9636-9647.

7. Graham HK, Holmes DF, Watson RB, Kadler KE. Identification of Collagen fibril fusion during vertebrate tendon morphogenesis. The process relies on unipolar fibrils and is regulated by collagen-proteoglycan interaction. J Mol Biol. 1999;295:891-902.

8. Rosa-Pimentel E, Sommarin Y, Wendel M, Oldberg Å, 
Heinegård D. Cartilage matrix proteins. An acidic oligomeric protein (COMP) detected only in cartilage. J Biol Chem. 1999;267:6132-6136.

9. Di Cesare P, Hauser N, Lehman D, Pasumarti S, Paulsson M. Cartilage oligomeric matrix protein (COMP) is an abundant component of tendon. FEBS Lett. 1994;354:237-240.

10. Mörgelin M, Heinegård D, Engel J, Electron PM. Microscopy of native cartilage oligomeric matrix protein purified from the swarm rat chondrosarcoma reveals a five-armed structure. J Biol Chem. 1992;267:6137-6141.

11. Oldberg $A$, Antonsson $P$, Lindblom $K$, Heinegård D. COMP (cartilage oligomeric matrix protein) is structurally related to the thrombospondins. J Biol Chem. 1992;267:22346-22350.

12. Holden P, Meadows RS, Chapman KL, Grant ME, Kadler KE, Briggs MD. Cartilage oligomeric matrix protein interacts with type IX collagen, and disruptions to these interactions identify a pathogenetic mechanism in a bone dysplasia family. J Biol Chem. 2001;276:6046-6055.

13. Rosenberg $\mathrm{K}$, Olsson $\mathrm{H}$, Mörgelin $\mathrm{M}$, Heinegård $\mathrm{D}$. Cartilage oligomeric matrix protein shows high affinity zinc-dependent interaction with triple helical collagen. J Biol Chem. 1998;273: 20397-20403.

14. Halász K, Kassner A, Mörgelin M, Heinegård D. COMP acts as a catalyst in collagen fibrillogenesis. J Biol Chem. 2007;282: 31166-31173.

15. Sodersten F, Hultenby K, Heinegard D, Johnston C, Ekman S. Immunolocalization of collagens (I and III) and cartilage oligomeric matrix protein (COMP) in the normal and injured equine superficial digital flexor tendon. Connect Tissue Res. 2013;54,62-66.

16. Lima FR, Trentin AG, Rosenthal D, Chagas C, Moura Neto $\mathrm{V}$. Thyroid hormone induces protein secretion and morphological changes in astroglial cells with an increase in expression of glial fibrillary acidic protein. J Endocrinol. 1997;154(1):167-175.

17. Trentin AG, Gomes FC, Lima FR, Neto VM. Thyroid hormone acting on astrocytes in culture. In Vitro Cell Dev Biol Anim. 1998;34(4):280-282.

18. Trentin AG. Thyroid hormone and astrocyte morphogenesis. J Endocrinol. 2006;189(2):189-197.

19. Farwell AP, Dubord-Tomasetti SA. Thyroid hormone regulates the extracellular organization of laminin on astrocytes. Endocrinology. 1999;140(11):5014-5021.

20. Farwell AP, Dubord-Tomasetti SA. Thyroid hormone regulates the expression of laminin in the developing rat cerebellum. Endocrinology. 1999;140(9):4221-4227.

21. Calloni GW, Alvarez-Silva M, Vituri C, Trentin AG. Thyroid hormone deficiency alters extracellular matrix protein expression in rat brain. Brain Res Dev Brain Res. 2001; 126(1):121-124.

22. Martinez R, Gomes FC. Neuritogenesis induced by thyroid hormone-treated astrocytes is mediated by epidermal growth factor/mitogen-activated protein kinase-phosphatidylinositol 3-kinase pathways and involves modulation of extracellular matrix proteins. J Biol Chem. 2002;277(51): 49311-49318.

23. Mendes-de-Aguiar CB, Costa-Silva B, Alvarez-Silva M, Tasca $\mathrm{Cl}$, Trentin AG. Thyroid hormone mediates syndecan expression in rat neonatal cerebellum. Cell Mol Neurobiol. 2008; 28(6):795-801.

24. Dezonne RS, Stipursky J, Araujo AP, et al. Thyroid hormone treated astrocytes induce maturation of cerebral cortical neurons through modulation of proteoglycan levels. Front Cell Neurosci. 2013;7:125.

25. Oliva F, Berardi AC, Misiti S, Falzacappa CV, lacone A, Maffulli N. Thyroid hormones enhance growth and counteract apoptosis in human tenocytes isolated from rotator cuff tendons. Cell Death and Disease. 2013;4, e705.

26. Magnan B, Bondi M, Pierantoni S, Samaila E. The pathogene- sis of Achilles tendinopathy. A systemic review. Foot Ankle Surg. 2014; Sep;20(3):154-159.

27. Purnell DC, Daly DD, Lipscomb PR. Carpal-tunnel syndrome associated with myxedema. Arch Intern Med. 1961;108:751756.

28. Garrity JA, Bahn RS. Pathogenesis of graves ophthalmopathy: implications for prediction, prevention, and treatment. Am J Ophthalmol. 2006;142:147-153.

29. Salvi M, Girasole G, Pedrazzoni M, et al. Increased serum concentrations of interleukin-6 (IL-6) and soluble IL-6 receptor in patients with Graves' disease. J Clin Endocrinol Metab. 1996;81:2976-2979.

30. Diez JJ, Hernanz A, Medina S, Bayon C, Iglesias P. Serum concentrations of tumour necrosis factor-alpha (TNF-alpha) and soluble TNF-alpha receptor p55 in patients with hypothyroidism and hyperthyroidism before and after normalization of thyroid function. Clin Endocrinol. (Oxf) 2002;57:515-521.

31. Yao L, Bestwick CS, Bestwick LA, Maffulli N, Aspden RM. Phenotypic drift in human tenocyte culture. Tissue Eng. 2006;12(7):1843-1849.

32. Lopez-De Leon A, Rojkind M. A simple micromethod for collagen and total protein determination in formalin-fixed paraffinembedded sections. J Histochem Cytochem. 1985; 33(8):737743.

33. Alcain FJ, Buron MI. Ascorbate on cell growth and differentiation. J Bioenerg biomembr. 1994;26(4):393-398.

34. Murad S, Grove D, Lindberg KA, Reynolds G, Sivarajah A, Pinnell SR. Regulation of collagen synthesis by ascorbic acid. Proc Natl Acad Sci USA 1981;78(5):2879-2882.

35. Birch HL, Thorpe CT, Rumian AP. Specialisation of extracellular matrix for function in tendons and ligaments. Muscles Ligaments Tendons J. 2013;21;3(1):12-22.

36. Padulo J, Oliva F, Frizziero A, Maffulli N. Muscles, Ligaments and Tendons Journal. Basic principles and recommendations in clinical and field science research. MLTJ. 2013;4:250-252.

37. de Rycker C, Vandalem JL, Hennen G. Effects of 3,5,3'-triiodothyronine on collagen synthesis by cultured human skinfibroblasts. FEBS Lett. 1984;174(1):34-37.

38. Yao J, Eghbali M. Decrease collagen gene expression and absence of fibrosis in thyroid hormone-induced myocardial hypertrophy. Response of cardiac fibroblasts to thyroid hormone in vitro. Circ Res. 1992;71(4):831-839.

39. Oliva F, Gatti S, Porcellini G, Forsyth NR, Maffulli N. Growth factors and tendon healing. Med Sport Sci. 2012;57:53-64.

40. Maffulli N, Ewen SW, Waterston SW, Reaper J, Barrass V. Tenocytes from ruptured and tendinopathic achilles tendons produce greater quantities of type III collagen than tenocytes from normal achilles tendons. An in vitro model of human tendon healing. Am J Sports Med. 2000;28(4):499505.

41. Vesentini S, Redaelli A, Gautieri A. Nanomechanics of collagen microfibrils. Muscles Ligaments Tendons J. 2013;3(1):23-34.

42. Giai Via A, De Cupis M, Spoliti M, Oliva F. Clinical and biological aspects of rotator cuff tears. Muscles Ligaments Tendons J. 2013;3(2):70-79. Erratum in: Muscles Ligaments Tendons J. 2014;3(4):359.

43. Derwin KA, Soslowsky LJ. A quantitative investigation of structure-function relationships in a tendon fascicle model. J Biomech Eng. 1999;121(6):598-604.

44. Derwin KA, Soslowsky LJ, Kimura JH, Plaas AH. Proteoglycans and glycosaminoglycan fine structure in the mouse tail tendon fascicle. J Orthop Res. 2001;19(2):269-277.

45. Lin TW, Cardenas L, Soslowsky LJ. Biomechanics of tendon injury and repair. J Biochem. 2004;37(6):865-877.

46. Matuszewski PE, Chen YL, Szczesny SE, Lake SP, Elliott DM, Soslowsky LJ, Dodge GR. Regional variation in human supraspinatus tendon proteoglycans: decorin, biglycan, and aggrecan. Connect Tissue Res. 2012;53(5):343-348. 
47. Das B, Cai L, Carter MG, Piao YL, Sharov AA, Ko MS, Brown DD. Gene expression changes at metamorphosis induced by thyroid hormone in Xenopus laevis tadpoles. Dev Biol. 2006; 291(2):342-355.

48. Adams JC, Lawler J. The thrombospondins. Cold Spring Harb Perspect Biol. 2011; 3(10):a009712.

49. Mackie EJ, Tatarczuch L, Mirams M. The skeleton: a multifunctional complex organ: the growth platechondrocyte and endochondral ossification. J Endocrinol. 2011;211(2):109.

50. Rock MJ, Holden P, Horton WA, Cohn DH. Cartilage oligomer- ic matrix protein promotescell attachment via two independent mechanisms involving CD47 and alphaVbeta3 integrin. Mol Cell Biochem. 2010;338(1-2):215-224.

51. Jordan JM. Cartilage oligomeric matrix protein as a marker of osteoarthritis. J Rheumatol Suppl. 2004;70:45-49.

52. Dakin SG, Smith RK, Heinegård D, Önnerfjord P, Khabut A, Dudhia J. Proteomic analysis of tendon extracellular matrix reveals disease stage-specific fragmentation and differential cleavage of COMP (cartilage oligomeric matrix protein). J Bio Chem. 2014;289(8):4919-4927. 